\title{
Misceléanea
}
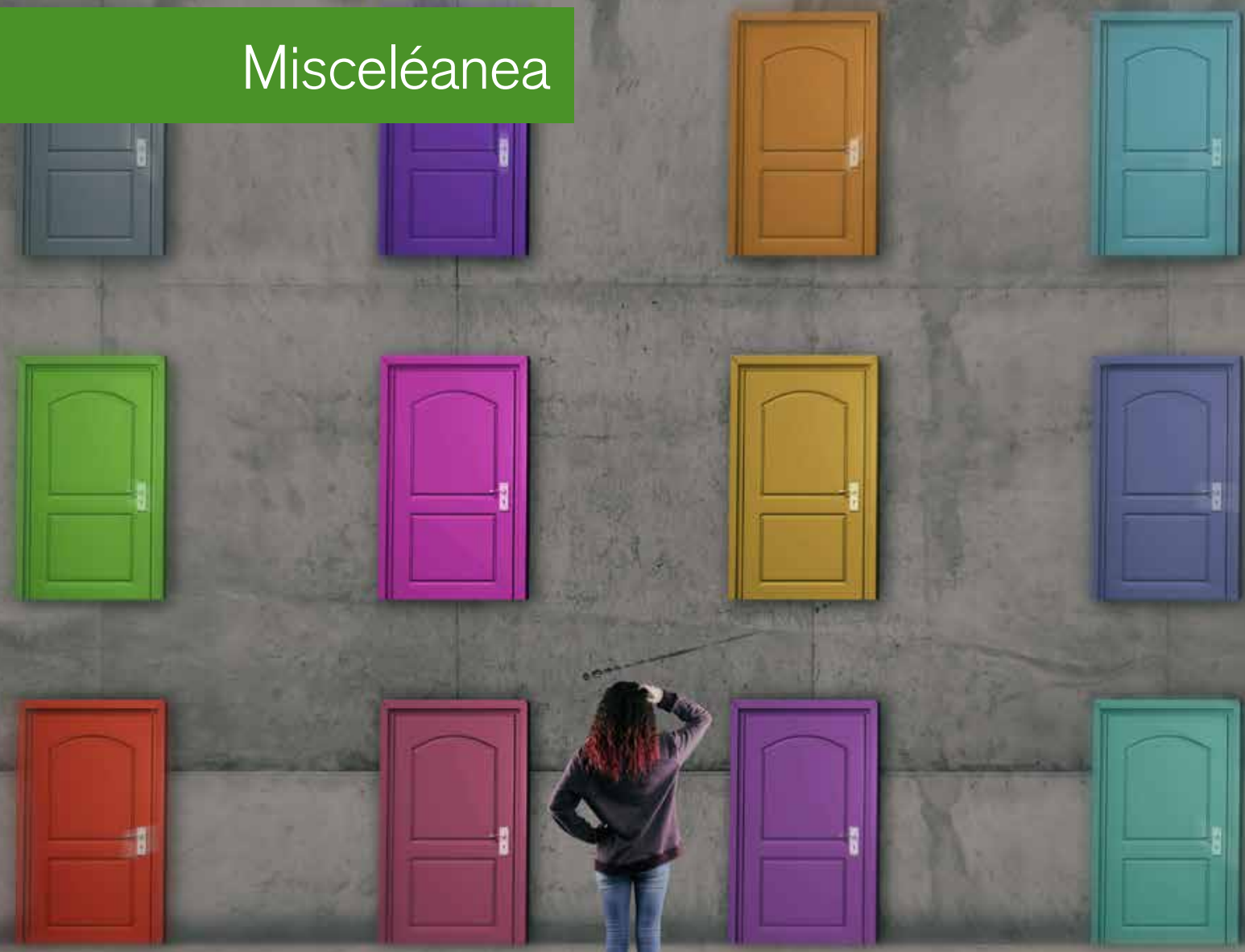

\section{Inteligencia emocional y toma de decisiones bioéticas}

Emotional Intelligence and Bioethics Decision Making 
Resumen

Abstract

Key words
La inteligencia emocional es un concepto que ha ido adquiriendo mayor protagonismo en los últimos años. De este modo, se ha investigado su influencia y aplicación en distintos procesos, por ejemplo, en la toma de decisiones. Las decisiones bioéticas personales conllevan emociones de gran intensidad, por lo que la inteligencia emocional se torna indispensable con el fin de realizar una correcta gestión del proceso emocional y, de esta manera, ser lo más libres y autónomos posible en nuestras decisiones.

The concept of emotional intelligence has gained prominence in recent years. Thus, its influence and application in different processes such as decision making, have been investigated. Personal bioethical decisions involve emotions of great intensity, therefore emotional intelligence becomes an indispensable tool for us to correctly manage the emotional process and thus be as free and autonomous as possible in our decisions.

Inteligencia emocional; toma de decisiones; bioética.

Emotional intelligence; decision making; bioethics.

Recibido: 25/07/2020. Aceptado: 01/05/2021 
En una sociedad en la que los cambios técnicos y científicos tienen lugar a una mayor velocidad cada día, la complejidad que este desarrollo ha traído a nuestras vidas se traslada, por consiguiente, también a los problemas bioéticos. Hasta hace pocos años, el abordaje de los debates éticos se daba exclusivamente en un plano intelectual, identificándose este de manera restrictiva con lo puramente racional. Actualmente, y gracias al desarrollo de la psicología, se ha comenzado a tener más presente la importancia del papel que las emociones juegan en la toma de decisiones. La inteligencia emocional supone la toma de consciencia de esta dimensión afectiva, lo cual nos permite tomar decisiones en el plano bioético con mayor libertad y autonomía.

\section{1. ¿Qué es la inteligencia emocional?}

La inteligencia emocional es un constructo psicológico y, como otros muchos, no es fácil de conceptualizar, ya que cuenta con distintas definiciones, dependiendo de cada autor y la formación de los mismos. A continuación, es preciso

La inteligencia emocional es

un concepto relativamente

novedoso que ha ido

adquiriendo mayor

protagonismo en los últimos

años, de este modo, podría ser

interesante prestar atención a

los precursores y antecedentes mencionar las definiciones de dos autores con gran influencia y prestigio en la materia. En primer lugar, en 1995 Daniel Goleman publica su célebre libro Inteligencia Emocional, en el cual define la inteligencia emocional como la "capacidad para reconocer nuestros propios sentimientos y los de los demás, para motivarse y gestionar la emocionalidad en nosotros mismos y en las relaciones interpersonales". En segundo lugar, en el año 2000, Salovey y Mayer definen la inteligencia emocional como "la capacidad de procesar la información emocional con exactitud y eficacia, incluyéndose la capacidad para percibir, asimilar, comprender y regular las emociones" (García-Fernández \& GiménezMas, 2010). A través de ambas definiciones, se puede observar cómo la inteligencia emocional desempeña un papel fundamental en la gestión de las propias emociones $y$, al mismo tiempo, en las emociones del otro.

La inteligencia emocional es un concepto relativamente novedoso que ha ido adquiriendo mayor protagonismo en los últimos años, de este modo, podría ser interesante prestar atención a los precursores y antecedentes de la inteligencia emocional. Pues bien, el principal precursor de la inteligencia emocional llega en 1983 con la publicación del libro Frames of Mind de Howard Gardner. En este libro, el autor plantea por primera vez el término de inteligencias múltiples. Dentro de estas inteligencias existen concretamente dos que suponen el inicio de la inteligencia emocional: la inteligencia interpersonal y la inteligencia intrapersonal. Por un lado, la inteligencia interpersonal hace referencia a la parte del pensamiento que nos permite ponernos en el lugar del otro, comprender sus percepciones y relacionarnos con el mundo físico que nos rodea. Por otro lado, la inteligencia intrapersonal supone la parte del pensamiento orientada hacia dentro, hacia uno mismo, permitiéndonos así desenvolvernos de forma eficaz en la vida (Moreno, Vicente, \& Martínez, 1998). Posteriormente, Daniel Goleman unifica ambas inteligencias y desarrolla el concepto "inteligencia emocio- 
nal" que resalta la importancia de las propias emociones, así como de las emociones de quien tenemos en frente.

Continuando con la contextualización del término, cabría mencionar los dos macromodelos principales que existen en inteligencia emocional. En primer lugar, los modelos mixtos son aquellos que se centran en la relación existente entre la personalidad y la inteligencia emocional. Dentro de este macromodelo se encuadra el modelo de inteligencia emocional propuesto por Daniel Goleman. En España, es el modelo mixto de inteligencia emocional el que ha gozado de mayor éxito y aplicación en el ámbito edu-

En un principio, la inteligencia

ha estado más enfocada al

plano racional del hombre;

sin embargo, en la actualidad

se comprende al ser humano

desde una visión integradora

que reconoce el papel

fundamental de las emociones

en nuestra vida cativo. En segundo lugar, los modelos de habilidades se centran en cómo se utiliza la inteligencia emocional en el aprendizaje. Dentro de este macromodelo se ubica el modelo de Salovey y Mayer (García-Fernández \& Giménez-Mas, 2010).

Una vez aclarada la definición de inteligencia emocional, se puede abordar su influencia en la toma de decisiones. La inteligencia emocional es un término que ha evolucionado notablemente en los últimos años. En un principio, la inteligencia ha estado más enfocada al plano racional del hombre; sin embargo, en la actualidad se comprende al ser humano desde una visión integradora que reconoce el papel fundamental de las emociones en nuestra vida. La inteligencia emocional es un concepto que se sustenta en los términos previos de inteligencia y emoción y, de este modo, queda patente la necesidad de prestar atención a la relevancia de las emociones en el ser humano y a la importancia de un término que dé cabida tanto a la razón como a la emoción (Leal \& Tutorial, 2011).

\section{Inteligencia emocional y el proceso de toma de decisiones}

Los seres humanos somos nuestras decisiones y nuestras decisiones son fruto de lo que sentimos y amamos. A diario, tomamos un gran número de decisiones, algunas incluso de manera automática como ¿qué voy a desayunar hoy? Sin embargo, el ser humano, a lo largo de su vida, también se enfrenta a decisiones con gran transcendencia, como ¿quiero ser madre? 0 ¿quiero recibir un tratamiento de quimioterapia? Cabe mencionar que las decisiones bioéticas se enmarcarían dentro del último grupo y es sencillo reconocer la carga emocional que suponen. Por ello, es fundamental tener presente la importancia de las emociones en el ser humano y el papel tan crucial que desempeñan en el proceso decisorio. Este hecho ofrece una nueva forma de entender cómo se enfrenta el ser humano a la toma de decisiones importantes.

A lo largo de la historia, el proceso de toma de decisiones ha evolucionado considerablemente. En un comienzo, la toma de decisiones estaba basada completamente en la racionalidad del hombre y se premiaban aquellas elecciones tomadas sobre la base de la razón. El estudio de la toma de decisión tiene su origen en el campo de las matemáticas, en los siglos XVII y XVIII, y paulatinamente se va extendiendo a la economía y la psicolo- 
gía. A través de la psicología, se comienza a incluir en el proceso decisorio fenómenos como la atención, las expectativas o la memoria que no habían sido tenidos en cuenta hasta la fecha. Del mismo modo, empiezan a introducirse los sesgos y heurísticos, lo que permite romper con la concepción clásica

A mediados del siglo XIX, se despierta un gran afán por el estudio de los mecanismos neurológicos que permiten a los seres humanos tomar decisiones que contemplaba únicamente la razón para descubrir el papel que desempeñan las emociones en la toma de decisión (León, 1987).

A mediados del siglo XIX, se despierta un gran afán por el estudio de los mecanismos neurológicos que permiten a los seres humanos tomar decisiones. Este interés se debió a casos notorios como el de Phineas Cage, un hombre al que tras un accidente de trabajo se le dañó la corteza prefrontal y, aunque no presentaba daños a nivel de operaciones mentales, tanto su personalidad como su comportamiento se vieron afectados y perjudicados.

Así pues, a través del uso de tareas específicas para el análisis de la toma de decisión y el posterior desarrollo de técnicas de neuroimagen, se han observado las siguientes áreas cerebrales como claves a la hora de tomar decisiones (Martínez-Selva, SánchezNavarro, Bechara, \& Román, 2006; Rivera \& Flórez, 2017):

- La corteza prefrontal ventromedial: se encarga de la elaboración e integración de todos aquellos factores que se ven implicados en la toma de decisiones. Esta área cerebral es responsable de combinar la información sensorial, los estados somáticos y las experiencias anteriores y, por ello, está relacionada con la evaluación de las consecuencias de las decisiones a largo plazo. Además, se ha percibido que la corteza prefrontal ventromedial presenta mayor implicación que la izquierda en la conducta social, las emociones y la toma de decisiones.

- La corteza prefrontal dorsolateral: está estrechamente relacionada con las funciones ejecutivas y atencionales, lo que permite la integración de los datos sensoriales. En consonancia, se ha observado que las lesiones en la corteza prefrontal dorsolateral suponen problemas en la toma de decisiones, debido especialmente a la memoria de trabajo y la retención de información, lo que desencadena elecciones desventajosas.

- La amígdala cerebral: en ella tienen lugar las conexiones entre los estímulos y sus consecuencias aversivas, lo cual repercute en la posterior toma de decisiones. Esta área cerebral tiene una fuerte asociación con la emoción del miedo y, además, juega un papel fundamental en las respuestas emocionales. Las lesiones en la amígdala impiden a los individuos experimentar las emociones de las situaciones afectivas, lo que obstaculiza la valoración de consecuencias futuras.

- La corteza cingulada anterior: se encarga de la regulación de la propia conducta, donde se incluye el proceso de inhibición de conducta. Además, se ha percibido una mayor actividad de la corteza cingulada anterior en consecuencias negativas, situaciones de incongruencia o en toma de decisiones riesgosas o de incertidumbre. Del mismo modo, esta área cerebral participa en la selección de conductas adecuadas, en la detección del error y en los cálculos sobre las probabilidades de recompensa.

A continuación, siguiendo con la contextualización del proceso decisorio cabría destacar algunas de las teorías de la decisión con mayor relevancia: 
En primer lugar, la teoría de la utilidad afirma que los sujetos racionales son aquellos individuos que buscan las máximas utilidades. Desde este modo, se plantean tres tipos de contextos en los que tomar decisiones: ciertos, riesgosos e inciertos. En contextos de certidumbre, el resultado de todas las acciones es totalmente predecible; en contextos de riesgo, la probabilidad que resulta de los distintos resultados es conocida (elección cara o cruz de una moneda); y en contextos de incer-

Damasio postula que

la experiencia previa va

provocando una respuesta

en el cuerpo, la cual queda

grabada en la memoria. Esta

respuesta puede ser consciente

o inconsciente, y retorna en

circunstancias similares

tidumbre, las probabilidades son parcial o totalmente desconocidas (Santiago \& Cante, 2009). Dentro de esta teoría, existe la variación de la teoría de la utilidad subjetiva, que incluye el valor subjetivo de cada individuo en la toma de decisiones. Respecto de esta variación, podría establecerse una relación con el paradigma utilitarista en bioética. Dicho paradigma propone "el mayor bien para el mayor número de personas" y la teoría subjetiva de la utilidad introduce los intereses propios de cada individuo al tomar decisiones. De esta manera, es importante tener en cuenta que las decisiones bioéticas no suelen ser decisiones certeras $y$, por ello, se incluyen los hechos objetivables y los valores subjetivos de las personas involucradas.

En segundo lugar, la teoría de la decisión racional postula que la decisión más racional es aquella que proporciona mayores beneficios para quien toma la decisión y, además, persigue tomar decisiones correctas en situaciones de incertidumbre (González Bonome, 2010). De aquí la relación existente entre la teoría de la decisión racional y la bioética. La toma de decisiones bioéticas se suele dar en condiciones de incertidumbre e implica un continuo manejo de valores, lo que puede volver más complejo el proceso decisorio. Pues bien, según la teoría de la decisión racional en bioética la mejor opción es aquella que suponga el máximo beneficio posible a pesar de la incertidumbre.

Por último, la teoría del marcador somático propuesta en 1994 por Antonio Damasio. Esta teoría supone un gran avance, ya que rompe con el dualismo cerebro-cuerpo y se plantea el concepto de "unidad corporal". Por primera vez, se propone que el proceso de toma de decisiones se ve dirigido por señales emocionales relacionadas con la homeostasis, los sentimientos y las emociones. Damasio postula que la experiencia previa va provocando una respuesta en el cuerpo, la cual queda grabada en la memoria. Esta respuesta puede ser consciente o inconsciente, y retorna en circunstancias similares. Además los marcadores somáticos presentan valencias tanto positivas como negativas, por lo que si se asocian a un resultado positivo, actúan como detonadores de la acción; mientras que si se asocian a un resultado negativo, actúan como inhibidores de la acción (Morandín-Ahuerma, 2019).

\section{Inteligencia emocional y bioética}

Antonio Damasio afirmó que "no somos máquinas pensantes que sentimos, somos máquinas sentimentales que pensamos" (2005). Esta máxima refleja que los seres humanos somos seres sintientes, lo que implica que las emociones son una parte intrín- 
seca del hombre y, por ello, lo propio no es ignorarlas, sino aceptarlas y apreciarlas. La gran mayoría de las veces, las decisiones bioéticas suponen una fuerte carga emocional, por lo tanto, aprender a identificarlas y manejarlas puede ser clave a la hora de tomar decisiones. Además, es importante comprender que el objetivo no es apartar las emociones de nosotros mismos, pues no es posible, sino más bien ofrecerles el lugar que merecen en cada uno de nosotros, aprendiendo a tomar la mejor decisión posible. En cuanto a decisiones complejas se refiere, las emociones desempeñan un papel fundamental, ya que suelen determinar nuestras preferencias de un modo más duradero. De esta manera, se plantea una relación entre las emociones y las preferencias, tanto a corto como a largo plazo, que impulsa la toma de decisiones del hombre. En la actualidad, es evidente que la emoción y la razón están estrechamente relacionadas y se ha

La relación existente entre

las emociones y la toma de decisiones es bidireccional, por tanto, las emociones influyen

en nuestras decisiones $y$, al

mismo tiempo, el resultado de estas decisiones tiene un gran impacto en las emociones que sentimos planteado que la inteligencia emocional podría considerarse el puente de unión entre ambas (Zubiría, 2007).

En cuanto a la inteligencia emocional y la toma de decisiones, en el año 2017 se lleva a cabo un experimento en aras de investigar la relación existente entre la inteligencia emocional y la toma de decisiones. Los datos obtenidos mostraron una correlación considerablemente alta $(r=.871)$ y estadísticamente significativa en 0.01 entre la inteligencia emocional y la toma de decisiones. Al mismo tiempo, se obtuvo una fuerte correlación $(r=.810)$ entre la estabilidad emocional y la toma de decisiones (Baba \& Siddiqi, 2017). Este dato es especialmente interesante, ya que refleja que existe una relación entre nuestro estado emocional y el proceso decisorio, por lo que la gestión emocional se torna indispensable. Además, es relevante tomar en cuenta la estadística ya que una buena fuente de datos supone la base de una buena teoría.

La relación existente entre las emociones y la toma de decisiones es bidireccional, por tanto, las emociones influyen en nuestras decisiones $y$, al mismo tiempo, el resultado de estas decisiones tiene un gran impacto en las emociones que sentimos. Un aspecto ampliamente estudiado y muy interesante desde el punto de vista bioético es la relación entre los resultados negativos y las emociones desencadenantes. Las dos emociones más comunes que emanan de un resultado negativo son el arrepentimiento y la desilusión. Asimismo, se ha observado que la mera anticipación de tales emociones puede influir en nuestra toma de decisiones y acciones (Schwarz, 2000). La relevancia bioética radica en el peso tan considerable que pueden tener los resultados negativos en un tratamiento, un embarazo o una situación compleja. Por ejemplo, en el caso de tratamientos fallidos es esperable que el paciente sienta desilusión, miedo, arrepentimiento, incertidumbre, etc., y estas emociones pueden influir de manera notoria en sus decisiones futuras, como recibir otro tratamiento o rechazarlo.

En consonancia con lo anterior, otro aspecto a resaltar es que los seres humanos tendemos a experimentar mayor arrepentimiento por los actos que cometemos que por aquellos otros que omitimos. La aplicabilidad de este hecho en bioética se observa en, por ejemplo, la problemática de las vacunas. En 1990 Ritov y Baron llevaron a cabo un experimento en el cual se mostraba que había padres que preferían no vacunar a sus 
hijos debido a las graves consecuencias que podía tener la vacuna, ignorando que las consecuencias de no poner la vacuna podía ser la muerte de sus hijos. Este es un claro ejemplo de cómo el sentimiento anticipado de arrepentimiento nos puede llevar a omitir determinadas acciones con gran repercusión. En estos casos, la inteligencia emocional permite identificar estas emociones anticipadas y actuar con mayor conciencia con el fin de elegir el mejor curso de acción posible (Sevdalis, Petrides, \& Harvey, 2007; Schwarz, 2000).

La investigación ha estudiado las diferencias entre hombres y mujeres en lo que a inteligencia emocional se refiere. De este modo, los resultados obtenidos han demostrado que las mujeres presentan superioridad en empatía y atención emocional, en tanto que

Así pues, los seres humanos

tendemos a escoger el curso

de acción que maximiza

nuestras emociones positivas

y disminuye las emociones

negativas los hombres muestran superioridad en regulación emocional. En la misma línea, se ha visto que las mujeres suelen presentar mayor capacidad para percibir sus emociones e incluso para comprenderlas y los hombres destacan en el control de impulsos y la tolerancia al estrés (Núñez, Fernández-Berrocal, Rodríguez, \& Postigo, 2008). Estas desemejanzas podrían deberse a las diferencias en los roles sociales entre hombres y mujeres, $y$ a la educación que puede ir acompañada de dichas diferencias. Tradicionalmente, las mujeres han sido las responsables del cuidado del hogar, de los hijos, etc., mientras que los hombres han podido recibir una educación más focalizada en disimular las emociones, ¿quién no ha escuchado alguna vez que "los hombres no lloran"?

Continuando con la influencia de la inteligencia emocional en la toma de decisiones bioéticas, es importante señalar que existen dos mecanismos fundamentales a través de los cuales las emociones influyen en el proceso decisorio.

En primer lugar, está el mecanismo de las "emociones esperadas", que son aquellas predicciones que realizamos sobre las consecuencias emocionales de una decisión. Cabe aclarar que no se trata de las emociones que se experimentan en el momento de la toma de decisión, sino de una predicción acerca de las emociones que surgirán en el sujeto a futuro, tras la elección de un curso de acción determinado. Así pues, los seres humanos tendemos a escoger el curso de acción que maximiza nuestras emociones positivas y disminuye las emociones negativas. En la toma de decisiones bioéticas personales, dichas emociones juegan un papel fundamental, ya que, por ejemplo, si antes de un tratamiento de quimioterapia el paciente predice que va a estar agotado, desanimado o triste, y se deja llevar por dichas emociones sin identificarlas ni elaborarlas, es muy probable que decida no recibir un segundo tratamiento. De este modo, la inteligencia emocional es clave para decisiones bioéticas tan cruciales que conllevan grandes consecuencias vitales (Loewenstein \& Lerner, 2003).

En segundo lugar, se encuentra el mecanismo de las "emociones inmediatas", que son aquellas que experimenta el sujeto decisorio en el mismo momento en el que debe tomar una decisión. A medida que las emociones se vuelven más intensas, aumenta su capacidad de influir en nuestro comportamiento y nuestros procesos cognitivos. En las cuestiones del campo de la bioética, la carga emocional suele ser considerablemente alta, lo que podría terminar dificultando el proceso de toma de decisión. Por lo tanto, 
la inteligencia emocional se vuelve indispensable a la hora de regular nuestras emociones, de manera que potencien la toma de decisiones, en lugar de dificultarla. Al mismo tiempo, la inteligencia emocional nos ayuda a tomar decisiones de forma más ventajosa y adaptativa, puesto que, como seres sintientes, no podemos apartar las emociones de nuestra vida cotidiana ni de nuestras decisiones (Loewenstein \& Lerner, 2003).

Las emociones son una parte innata del ser humano y como tal es preciso ofrecerles el espacio que merecen. De hecho, se ha llegado a decir que se es más fiel a uno mismo cuando se siente más profundamente y no cuando se piensa mejor. De este modo, lo verdaderamente profundo tiene más afinidad con lo sensible que con lo intelectual, por lo que una emoción corporal se registra en la memoria más sencilla y duraderamente que una opinión de la razón (Blasco, Moreto, Janaudis, de Benedetto, Marroquín, \& Altiseri, 2013). En relación con esto, el proceso cognitivo de recuperación de la información

Las emociones son una parte

innata del ser humano y como

tal es preciso ofrecerles el

espacio que merecen. De hecho,

se ha llegado a decir que se es

más fiel a uno mismo cuando se

siente más profundamente y no

cuando se piensa mejor ha sido ampliamente estudiado y se conoce que los seres humanos tendemos a recuperar la información congruente con la emoción que sentimos, independientemente de si dicha información no se relaciona con el momento que desencadenó la emoción. Este hecho tiene una aplicación bioética sustancial, ya que la investigación ha concluido que las emociones son un determinante primordial en la búsqueda de cuidado, en la adherencia y la recuperación del tratamiento (Salovey \& Birnbaum, 1989). De esta manera, los individuos que experimentan una intensa emoción de tristeza se van a enfrentar a la enfermedad desde una concepción más pesimista y sus decisiones también se verán influenciadas, por ejemplo, rechazando un trasplante o tratamiento. Por lo tanto, siendo conocedores de esta información somos capaces de prestar atención al papel tan positivo que puede desempeñar la inteligencia emocional en estos casos, ya que seremos capaces de reconocer nuestras emociones, en lugar de ignorarlas, tomando decisiones con mayor libertad y autonomía.

\section{Conclusiones}

El desarrollo de la inteligencia emocional ha permitido obtener una visión más integral del ser humano comprendiendo al mismo como un todo. Dentro de este "todo" se ha reconocido que el hombre no es solo razón, sino también emoción. Y lo que es aún más importante, se ha reconocido el papel tan fundamental que desempeñan las emociones en la toma de decisiones. En consonancia, es importante comprender que las emociones no son nuestro enemigo, por lo que no debemos reprimirlas, olvidarlas, rechazarlas o incluso castigarnos por experimentarlas. Las emociones no deben supeditarse a la razón del mismo modo que los seres humanos no deberíamos renegar de la capacidad de sentir, puesto que forma parte de nuestra propia naturaleza.

Las emociones ejercen una gran influencia en el proceso decisorio y, además, cuanto mayor sea la carga emocional, más compleja se vuelve la toma de decisiones. Por con- 
siguiente, el manejo emocional en bioética se convierte en un aspecto esencial a tener en cuenta. La inteligencia emocional persigue poner fin al debate entre razón y emoción a través de la integración de ambas y, de este modo, tomar decisiones de la forma más satisfactoria posible. Además, la inteligencia emocional no se limita al plano intrapersonal, sino que se expande al plano interpersonal, por lo tanto, abarca nuestras propias emociones, así como las de aquellos que nos rodean.

Finalmente, es preciso preguntarse ¿para qué la inteligencia emocional en bioética? Pues bien, la aplicación de la inteligencia emocional en el ámbito de la bioética podría suponer un gran avance y una herramienta de ayuda para pacientes y sanitarios. Por un lado, la inteligencia emocional nos posibilita un trabajo personal de introspección, el cual nos proporciona un mayor autoconocimiento sobre nosotros mismos. Dicho autoconocimiento podría ser clave para los pacientes, ya que se experimentan emociones de gran intensidad y el manejo de las mismas es necesario para tomar decisiones satisfactorias. Por otro lado, los sanitarios también podrían beneficiarse de la inteligencia emocional conociendo el impacto que tienen las emociones en el otro, en estrategias de comunicación y en la elaboración de sus propias emociones.

En conclusión, la inteligencia emocional en bioética podría ser un futuro e interesante campo de trabajo al que dirigir nuestra atención.

\section{Referencias}

Baba, M. M., \& Siddiqi, M. A. (2017). Emotional intelligence and decision making effectiveness: An empirical study of institutions of higher learning. Amity Global Business Review, 12, 81-89.

Blasco, P. G., Moreto, G., Janaudis, M. A., Benedetto, M. A. C. de, Marroquín, M. D., \& Altiseri, R. (2013). Educar las emociones para promover la formación ética. Persona y Bioética, 17(1), 28-48. https://doi.org/10.5294/ pebi.2013.17.1.2

Damasio, A. (2005). En busca de Spinoza. La neurobiología de la emoción y los sentimientos. Barcelona: Editorial Grupo Planeta.

Goleman, D. (2015). El cerebro y la inteligencia emocional: nuevos descubrimientos. B DE BOOKS.

González Bonome, M. (2010). La racionalidad en la toma de decisiones: Análisis de la teoría de la decisión de Herbert A. Simon. La Coruña: Netbiblio.

González, R. C., Aranda, D. R., \& Berrocal, P. F. (2010). Docentes emocionalmente inteligentes. Revista electrónica interuniversitaria de formación del profesorado, 13(1), 41-49.

Leal, A., \& Tutorial, O. Y. A. (2011). La inteligencia emocional. Digital Innovación y experiencias educativas, 39.

León, O. G. (1987). La toma de decisiones individuales con riesgo desde la psicología. Estudios de Psicología, 8(29-30), 79-94. https://doi.org/10.1080/02109395.1987.10821482

Loewenstein, G., \& Lerner, J. S. (2003). The role of affect in decision making. Handbook of affective science, 619(642), 3 . 
Martínez-Selva, J. M., Sánchez-Navarro, J. P., Bechara, A., \& Román, F. (2006). Mecanismos cerebrales de la toma de decisiones. Revista de neurología, 42(7), 411-418. https://doi.org/10.33588/rn.4207.2006161

Morandín-Ahuerma, F. (2019). La hipótesis del marcador somático y la neurobiología de las decisiones. Escritos de Psicología, 12(1), 20-29. https://doi.org/10.5231/psy.writ.2019.1909

Moreno, C. M., Vicente, E. S., \& Martínez, C. E. (1998). Revisión histórica del concepto de inteligencia: una aproximación a la inteligencia emocional. Revista latinoamericana de Psicología, 30(1), 11-30.

Núñez, M. T. S., Fernández-Berrocal, P., Rodríguez, J. M., \& Postigo, J. M. L. (2008). ¿Es la inteligencia emocional una cuestión de género? Socialización de las competencias emocionales en hombres y mujeres y sus implicaciones. Electronic journal of research in educational Psychology, 6(2), 455-474.

Rivera, L. F. S., \& Flórez, J. A. R. (2017). Bases neurales de la toma de decisiones e implicación de las emociones en el proceso. Revista Chilena de Neuropsicología, 12(2), 32-37.

Salovey, P., \& Birnbaum, D. (1989). Influence of mood on health-relevant cognitions. Journal of Personality and social Psychology, 57(3), 539. https://doi.org/10.1037/0022-3514.57.3.539

Santiago, J., \& Cante, F. (2009). Intuición, sesgos y heurísticas en la elección. Cuadernos de Economía, 28(50), 1-34.

Schwarz, N. (2000). Emotion, cognition, and decision making. Cognition \& Emotion, 14(4), 433-440. https://doi. org/10.1080/026999300402745

Sevdalis, N., Petrides, K. V., \& Harvey, N. (2007). Trait emotional intelligence and decision-related emotions. Personality and Individual Differences, 42(7), 1347-1358. https://doi.org/10.1016/j.paid.2006.10.012

Zubiría, S. (2007). Bioética, emociones e identidades. Revista colombiana de Bioética, 2(1), 115-132. 